\title{
Perioperative Hypertension Management during Facelift under Local Anesthesia with Intravenous Hypnotics
}

\author{
Ki Ho Chung ${ }^{1}$, Myeong Soo Cho' ${ }^{2}$, Hoon Jin ${ }^{3}$ \\ ${ }^{1}$ Jamie Aesthetic Plastic Surgery Clinic, Seoul; ${ }^{2}$ LIFT Aesthetic Plastic Surgery Clinic, Daegu; ${ }^{3}$ IDEA Plastic Surgery Clinic, Seoul, Korea
}

Perioperative hypertension is a phenomenon in which a surgical patient's blood pressure temporarily increases throughout the preoperative and postoperative periods and remains high until the patient's condition stabilizes. This phenomenon requires immediate treatment not only because it is observed in a majority of patients who are not diagnosed with high blood pressure, but also because occurs in patients with underlying essential hypertension who show a sharp increase in their blood pressure. The most common complication following facelift surgery is hematoma, and the most critical risk factor that causes hematoma is elevated systolic blood pressure. In general, a systolic blood pressure goal of $<150 \mathrm{~mm} \mathrm{Hg}$ and a diastolic blood pressure goal of $>65 \mathrm{~mm} \mathrm{Hg}$ are recommended. This article discusses the causes of increased blood pressure and the treatment methods for perioperative hypertension during the preoperative, intraoperative, and postoperative periods, in order to find ways to maintain normal blood pressure in patients during surgery. Further, in this paper, we review the causes of perioperative hypertension, such as anxiety, epinephrine, pain, and postoperative nausea and vomiting. The treatment methods for perioperative hypertension are analyzed according to the following 3 operative periods, with a review of the characteristics and interactions of each drug: preoperative antihypertensive medicine (atenolol, clonidine, and nifedipine), intraoperative intravenous (IV) hypnotics (propofol, midazolam, ketamine, and dexmedetomidine), and postoperative antiemetic medicine (metoclopramide and ondansetron). This article focuses on the knowledge necessary to safely apply local anesthesia with IV hypnotics during facelift surgery without the assistance of an anesthesiologist.

\section{Keywords Hypertension / Epinephrine / Hematoma / Clonidine / Propofol}

Received: 18 Apr 2017 • Revised: 4 Jun 2017 • Accepted: 5 Jun 2017

pISSN: 2234-6163 • elSSN: 2234-6171 • https://doi.org/10.5999/aps.2017.44.4.276 • Arch Plast Surg 2017;44:276-282
Correspondence: Ki Ho Chung Jamie Aesthetic Plastic Surgery Clinic, 3F, 136 Apgujeong-ro, Gangnam-gu, Seoul 06029, Korea

Tel: $+82-2-542-2399$

Fax: +82-2-542-7929

E-mail:yohammer@naver.com

This review article was presented at the Aesthetic Plastic Surgery 2016

Symposium of the Korean Society of Aesthetic Plastic Surgery on March 13 2016, in Seoul, Korea.

No potential conflict of interest relevant to this article was reported.

\section{INTRODUCTION}

Patients scheduled for surgery often show a temporary increase in their blood pressure throughout the preoperative and postoperative periods, and the pressure remains high until their condition stabilizes. This phenomenon is defined as perioperative hy- pertension. This phenomenon requires active treatment not only because it is observed in a majority of patients who are not diagnosed with high blood pressure, but also because it occurs in patients with underlying essential hypertension who tend to temporarily show a sharp increase or decrease in their blood pressure [1]. 
In this paper, we discuss perioperative hypertension identification and management, as well as the effects on blood pressure of drugs widely used in surgery. Plastic surgeons who perform facelifts under local anesthesia with intravenous (IV) hypnotics, a common clinical scenario, need to be fully aware of this fundamental information.

\section{CLINICAL SIGNIFICANCE OF PERIOPERATIVE HYPERTENSION}

Perioperative hypertension management in facelift patients holds clinical significance. Maintaining normal blood pressure is important for the following reasons: (1) It is the main factor affecting the patient's physical and mental stability during the preoperative period. (2) It reduces blood loss and stabilizes the patient's vital signs, while simultaneously providing a clear operative field to the surgeon, which eases the surgical process during the intraoperative period. (3) It minimizes bruising and swelling and results in a statistically significant decrease in the frequency of hematoma during the postoperative period [2].

The management of perioperative hypertension is critical, and an anesthesiologist can assist in the surgery using general anesthesia. However, in practice, a majority of facelift operations are conducted using a combination of local anesthesia and intravenous hypnotics, without an anesthesiologist's assistance. Therefore, the surgeon needs to fully comprehend the phenomenon of perioperative hypertension and effectively respond to it.

\section{TREATMENT GOALS FOR PERIOPERATIVE HYPERTENSION}

The prevention and treatment goals for transient perioperative hypertension are obviously different from the treatment goals for essential hypertension (Table 1). In case of essential hypertension, the treatment goal is to minimize cardiovascular complications over the long term, which requires focusing on improving patients' compliance with treatment.

Table 1. Treatment goals of hypertension
\begin{tabular}{|ll|}
\hline Essential hypertension patients' ideal blood pressure \\
$<140 / 90 \mathrm{~mm} \mathrm{Hg}$ & Uncomplicated HTN \\
$<130 / 80 \mathrm{~mm} \mathrm{Hg}$ & DM, CKD, CAD, LVH \\
$<125 / 75 \mathrm{~mm} \mathrm{Hg}$ & DM with microalbuminuria or nephropathy CKD or CAD \\
with proteinuria
\end{tabular}
$\begin{array}{ll}\text { Perioperative hypertension patiens' ideal blood pressure } \\
<150 / 65 \mathrm{~mm} \mathrm{Hg} & \text { Facelift }\end{array}$

HTN, hypertension; DM, diabetes mellitus; CKD, chronic kidney disease; CAD, coronary artery disease; LVH, left ventricular hypertrophy.
However, surgeons focus on only a few days before and after the operation because blood pressure only temporarily increases during this period and returns to normal thereafter. For cosmetic surgery, a subfield of plastic surgery, and particularly for facelift surgery, maintaining the systolic blood pressure at $<150$ $\mathrm{mm} \mathrm{Hg}$ and the diastolic blood pressure at $>65 \mathrm{~mm} \mathrm{Hg}$ is clinically important because considerable research has documented a statistically significant increase in the frequency of hematoma when the systolic blood pressure is higher than $150 \mathrm{~mm} \mathrm{Hg}$ $[3,4]$. Therefore, it is necessary to focus on and closely investigate the perioperative period for a few days when the surgeons observe the patients' blood pressure.

\section{UNDERSTANDING THE 3 PERIODS OF PERIOPERATIVE HYPERTENSION}

Perioperative hypertension can be divided into 3 periods relative to the procedure, and the reasons for increased blood pressure in each period need to be understood.

\section{Preoperative period}

The moment a patient perceives surgery as an imminent reality, his/her anxiety levels increase and endogenous adrenalin release is increased, which increases his/her blood pressure. Typically, the blood pressure reaches its climax starting on the morning of surgery to right before the operation.

\section{Intraoperative period}

Epinephrine, a commonly used local anesthetic, acts as exogenous adrenalin and increases blood pressure. Blood pressure can also increase due to pain or fear if the patient has not reached sufficient levels of sedation and calm.

\section{Postoperative period}

Blood pressure increases mainly due to an increase in endogenous adrenalin caused by pain in the surgical area or anxiety over the results of the surgery. Nausea and vomiting can worsen the situation.

\section{STRATEGIES FOR PERIOPERATIVE HYPERTENSION MANAGEMENT}

\section{Preoperative interview}

The surgeon needs to determine whether preventive medication is needed for perioperative hypertension during the first outpatient consultation. In general, the assessment should include the following: (1) Ask the patient about their daily blood 
pressure. (2) Observe the patient's psychological and emotional conditions during the consultation. (3) Directly ask about their fear or anxiety regarding surgery. (4) Ask about their recollection of a previous surgical experience and ask them the level of bruising or swelling during their recovery from the same. (5) Check their current blood pressure.

Having collected that information, the surgeon should assess whether preventive medication is necessary for the patient starting on the day of or the day before surgery. For example, a patient may claim that he/she has normal blood pressure while providing his/her medical history, but if that patient shows fear and anxiety regarding the operation and if the blood pressure taken at the end of the interview is high, the surgeon should conclude that the patient most likely has hospital hypertension and is very likely to show perioperative transient hypertension.

At this stage, the surgeon is confronted with the problem of not having a specific criterion or indication for preventive antihypertensive medication. This is because the extent of the autonomic nervous system reaction under the stress of surgery varies from person to person, and it is difficult to reach an informed conclusion from controlled studies when the medication for the perioperative period differs by the type of surgery and surgeon.

Despite this difficulty, all surgeons, including plastic surgeons, agree on the clinical significance of perioperative hypertension and the need for short-term treatment and for treating patients empirically $[5,6]$. As for facelift surgery, if anamnesis does not indicate an underlying disease such as syncope, orthostatic hypotension, or anemia, most plastic surgeons selectively administer preventive antihypertensive medication to patients showing normal or high blood pressure and many surgeons practice this as a routine procedure. Therefore, we need to understand the empirical treatment method that a majority of the surgeons prefer to conduct.

\section{Management of the preoperative period}

The currently used medications in the field of plastic surgery are as follows: 1) $\beta$-blockers, 2) a2 agonist drugs, and 3) calcium channel blockers (CCBs). The characteristics of these 3 types of medications can be summarized as follows:

\section{$\beta$-blockers}

$\beta$-blockers are competitive antagonists of endogenous adrenalin. They combine with $\beta 1$ receptors that are mostly located in the heart and reduce blood pressure by decreasing the heart rate and myocardial contractibility. They also combine with $\beta 2$ receptors, located mainly in the lungs, and contract the bronchiole, which increases airway resistance and decreases ventilation.

Therefore, when $\beta$-blockers are used in the perioperative peri- od, atenolol is mainly used, because it is a selective $\beta 1$ receptor blocker that selectively affects only the heart. In general, a single dose of $50 \mathrm{mg}$ is administered the day before or on the morning of surgery. Atenolol not only decreases blood pressure, but is also effective in the case of anxiety disorders. Indeed, $\beta$-blockers were first introduced and popularized by Hollywood celebrities who took the medication to treat stage fright that was accompanied by symptoms of a racing pulse and rapid breathing before they stood on stage. Considering the fact that one of the major causes of perioperative hypertension is anxiety, this characteristic of $\beta$-blockers is extremely attractive and when applied in actual clinical usage, the medical staff witness patients' emotional calm and a noticeable decrease in the risk of tachycardia.

However, $\beta$-blockers can cause bradycardia and conduction disorders; therefore, they should not be used in patients with sick sinus syndrome, atrioventricular block, conduction disorders, or bradycardia.

\section{$\alpha 2$ agonist drugs}

$\alpha 2$ receptors are localized in the central nervous system structures that control negative feedback within the autonomic nervous system. That is, when the sympathetic nervous system is active, the concentration of noradrenaline is increased. In response, $\alpha 2$ receptors are activated and inhibit the release of noradrenaline from nerve endings. Eventually, the overall sympathetic tone is reduced, reducing blood pressure and having a calming and pain-relieving effect.

In clinical practice, clonidine is a drug used for controlling perioperative hypertension [7]. A 0.1-mg oral tablet clonidine can be administered the day before or on the morning of surgery, or can be administered in the form of dermal patches.

Similar to $\beta$-blockers, $\alpha 2$ agonists should not be administered to patients with a history of syncope, orthostatic hypotension, and/or conduction disorders. Moreover, $\propto 2$ agonists must be gradually tapered off when discontinuing the medication, because a sudden discontinuation may cause rebound hypertension. The clonidine tablets that are commonly used during the perioperative period have an elimination half-life ranging from 12 to 16 hours, and in theory, caution for orthostatic hypotension is advised when the medication is still effective. Immediately after the medication is no longer effective, the patient needs to be monitored for rebound hypertension; however, the probability of rebound hypertension is extremely low when the drug is used for a short period of time.

\section{Calcium channel blockers}

CCBs, along with angiotensin-converting enzyme inhibitors and diuretics, are commonly used as primary treatment meth- 
ods for high blood pressure. CCBs widen blood vessels, which reduces the blood pressure rather rapidly. Among CCBs, nifedipine is the most appropriate agent for perioperative hypertension management. In South Korea, nifedipine is distributed in the forms of 5-mg or 10-mg soft capsules or 30-mg sustained-release preparations. The soft capsules have a shorter elimination half-life of 8 hours, while the sustained-release preparations of nifedipine have a longer half-life of 24 hours. This medication should be chosen on the basis of the type of surgery and patientspecific characteristics. However, in general, patients taking nifedipine for the first time exhibit a stable blood concentration on the second day after administration. Therefore, we recommend starting administration the day before surgery and additionally administering it as needed on the day of surgery. The appropriate dosage is $30 \mathrm{mg}$ a day.

The advantage of using nifedipine is the fast reduction of blood pressure. The disadvantages include dizziness in patients and frequent occurrences of reflex tachycardia. Furthermore, unlike the previously discussed $\alpha 2$ agonists and $\beta$-blockers, CCBs do not have anxiolytic effects. This is a tremendous disadvantage in using this medication, since an increase in the sympathetic tone caused by anxiety is a main cause of perioperative hypertension.

If the patient is not taking any other medication for blood pressure management, the surgeon needs to understand the characteristics of the 3 types of medications discussed above and administer the appropriate agent to manage perioperative hypertension. In general, a single dose is administered on the morning of or on the night before surgery.

For patients taking antihypertensive medication for underlying essential hypertension, the first obvious but important step to take is to consult with the prescribing cardiologist. After consultation, most patients are prescribed a temporarily increased dosage of their usual medication, most commonly angiotensinconverting enzyme inhibitors, diuretics, CCBs, or a combination thereof. This increased dosage is primarily the same as the treatment used for general high blood pressure in which first, the dosage is increased until the blood pressure reaches the desired range, and then, a new medication is added. The surgeon can increase the dosage on the basis of the consultation, and it is generally preferable to add an anxiolytic medication. It is important at this stage to check once again if the patient is taking any easily overlooked medication, such as low-dose aspirin, Oriental medicines, or herbs, and whether he/she has temporarily stopped taking those medications.

\section{Management of the intraoperative period}

Plastic surgeons need to have an understanding of the medicinal

\begin{tabular}{|c|c|c|c|c|}
\hline \multirow[b]{2}{*}{ IV hypnotics } & \multicolumn{2}{|c|}{$\begin{array}{l}\text { Cardiovascular } \\
\text { system }\end{array}$} & \multicolumn{2}{|c|}{$\begin{array}{l}\text { Respiratory } \\
\text { system }\end{array}$} \\
\hline & $\begin{array}{c}\text { Heart } \\
\text { rate }\end{array}$ & $\begin{array}{c}\text { Mean } \\
\text { arterial } \\
\text { pressure }\end{array}$ & Ventilation & Bronchodilation \\
\hline Propofol & $(-)$ & $\downarrow \downarrow \downarrow$ & $\downarrow \downarrow \downarrow$ & $(-)$ \\
\hline Midazolam & $(-)$ & $\downarrow \downarrow$ & $\downarrow \downarrow$ & $(-)$ \\
\hline Ketamine & $\uparrow \uparrow$ & $\uparrow \uparrow$ & $\downarrow$ & $\uparrow \uparrow \uparrow$ \\
\hline Dexmedetomidine & $\downarrow$ & $\downarrow \downarrow$ & $(-)$ & $(-)$ \\
\hline
\end{tabular}

drugs used during surgery that affect blood pressure. Among these drugs, IV hypnotics (Table 2), and epinephrine and anticholinergics that are combined with local anesthetics are clinically significant. In the following section, we will examine these drugs more closely.

\section{Propofol}

Propofol is the IV agent of choice for outpatient surgery because it is a short-acting medication that can quickly sedate or wake a patient: within 1 minute of the intravenous injection of 1.5-3.0 $\mathrm{mg} / \mathrm{kg}$ of propofol, the dose for induction of anesthesia, the patient loses consciousness for 5-10 minutes. It is not associated with hangovers or nausea and vomiting when it wears off, which makes it a preferred medication for cosmetic surgery. With these advantages, propofol has become used widely and has recently been considered a social problem, but these issues only arose due to the improper storage, reuse, and abuse of the medication. Propofol is still the safest drug with the least side effects among the currently available hypnotics. Propofol decreases the mean arterial pressure and causes respiratory depression. Although the decrease in blood pressure rarely causes a significant problem, respiratory depression should be carefully monitored. Furthermore, it is important to remember that propofol has no analgesic effect, which is a disadvantage.

\section{Midazolam}

Midazolam is commonly used for outpatient surgery and procedural sedations. Midazolam can be administered in all formsas an oral medication, intramuscular injection, or intravenous injection-depending on the surgeon's preference. When used as treatment before surgery, it is most advantageous for its qualities of decreasing the patient's anxiety levels while having an anterograde amnesia effect. Further, it is effective in the acute management of seizures. Midazolam reduces blood pressure and causes mild respiratory depression, but it is very rare to see severe clinical side effects when doses of under $5 \mathrm{mg}$ are used 
for preoperative treatment. Flumazenil, a midazolam antagonist, can be prepared for emergencies. As with propofol, midazolam has no analgesic effect.

\section{Ketamine}

Ketamine is a type of hypnotic that has anesthetic, amnesic, and dissociative effects and is commonly used in outpatient surgery. Ketamine is administered as an intramuscular or intravenous injection, and its biggest advantage is its analgesic effect. Ketamine has different effects on the cardiovascular system and respiratory system as compared to the other hypnotics. The heart rate and the mean arterial pressure are increased, and bronchodilation is induced, meaning that respiratory depression episodes are reduced and a relatively normal respiratory reflex pattern is maintained. However, visual disturbances, hallucinations, and delirium may be experienced during the recovery phase, and there is a risk of nausea and vomiting.

\section{Dexmedetomidine}

Dexmedetomidine is a highly selective $\alpha 2$ agonist that decreases the overall sympathetic tone by controlling negative feedback within the autonomic nervous system. As such, it has anxiolytic, sedative, and analgesic effects. The advantage of using this medication is that although the heart rate and the mean arterial pressure decrease, no respiratory depression occurs. Originally used for intensive care unit sedations, it is now used in outpatient surgery under local anesthesia for conscious sedation. Although it is effective in calming patients, relieving pain, and reducing blood pressure, extra attention must be paid to prevent syncope caused by hypotension and bradycardia, which may occur in cases without much postoperative recovery time.

\section{Epinephrine}

Most plastic surgeons use a mix of lidocaine and epinephrine as a local anesthetic because epinephrine reduces the toxicity of lidocaine and provides a clear operative field by causing local vasoconstriction. However, as local vasodilation occurs over time, epinephrine, which was once concentrated in the operation area, is absorbed into the systemic circulation. In the case of plastic surgery, the absorption peak occurs after 4-6 hours [8]. Epinephrine that is absorbed into the systemic circulation increases the patient's heart rate and blood pressure; therefore, minimizing its use during an operation is beneficial for postoperative blood pressure management [9]. Given the need to balance the benefits of epinephrine (that it minimizes bleeding by causing local vasoconstriction) with its disadvantage of increasing bleeding due to the increased blood pressure after surgery, the surgeon needs to empirically find the ideal epinephrine dilu- tion ratio and the total amount to be used per hour $[5,6,9]$.

\section{Management of the postoperative period}

Patients gradually recover consciousness in the recovery room after surgery. Patients' blood pressure may be elevated due to anxiety regarding the results of the operation, pain in the surgical area, and the influence of the epinephrine that was used during the surgery. Increased blood pressure due to anxiety and epinephrine is most likely already controlled with preoperative antihypertensive medications. The actual care needed during the recovery time is the prevention of pain, nausea, and vomiting.

Analgesics used generally in the field of plastic surgery are categorized into nonsteroidal anti-inflammatory drugs (NSAIDs), such as diclofenac or ketorolac, and opioids that have stronger analgesic effects. NSAIDs are generally sufficient for facelift surgery because the postoperative pain is minimal.

Postoperative nausea and vomiting frequently occur after IV hypnotic administration. Preventing these symptoms is an important factor in maintaining normotensive blood pressure, as well as in increasing the level of patients' satisfaction with their postoperative medical condition. The predisposing factors include being a young female, being a nonsmoker, motion sickness, and a long operation time. Postoperative nausea and vomiting commonly occur after forehead-lift surgery.

Prophylactic efforts are more important than the direct treatment of postoperative nausea and vomiting. Among the IV hypnotics, propofol should be chosen over the other medications because it prevents nausea and vomiting. However, ketamine and opioids should be used only if necessary, and in minimal doses, because they increase the incidence of nausea and vomiting. The most common prophylactic medicines used for nausea and vomiting are metoclopramide and ondansetron. Metoclopramide is a dopamine receptor antagonist that helps in emptying the gastrointestinal tract. Ondansetron is a 5-HT3 receptor antagonist that is used prior to chemotherapy. Although ondansetron has stronger antiemetic effects than metoclopramide, it is safe to administer them together because their mechanisms of action are different.

\section{DISCUSSION}

The most common complication of facelift surgery is hematoma. The risk factors for hematoma are systolic blood pressure, male sex, aspirin, and smoking, and among these risk factors, the intraoperative systolic blood pressure has a particularly strong correlation to hematoma. Furthermore, hematoma incidence increases when conducting neck-lift (neck dissection) surgery, whereas the dissection plane in facelifts is not related to 
hematoma $[2-4,9]$. If risk factors are confirmed to be present, medication that could cause bleeding tendencies in patients needs to be restricted in the preoperative period. The normal procedure is to determine the dissection plane and range after patients discontinue smoking. These are certain fundamental and invariable approaches to surgical management. Among the risk factors that may have a different prognosis depending on the surgeon's experience and interests is whether the systolic blood pressure is maintained at $<150 \mathrm{~mm} \mathrm{Hg}$ and the diastolic blood pressure at $>65 \mathrm{~mm} \mathrm{Hg}$.

Internationally renowned plastic surgeons with extensive experience in facelift surgery, such as D.C. Baker, F.E. Barton, J.M. Stuzin, and R.J. Rohrich, along with South Korean plastic surgeons, administer preoperative medication to manage perioperative hypertension [8]. This article reviewed 3 oral medications that can be used in South Korea ( $\beta$-blockers, $\alpha 2$ agonists, and CCBs). The first concern that arises in clinical practice is the selection of medication, and a fundamental understanding of the representative receptors in the sympathetic nervous system is necessary.

a1 receptors are mainly located in the blood vessels and cause vasoconstriction, while $\alpha 2$ receptors are localized in the central nervous system structures that control negative feedback and inhibit the release of noradrenaline from sympathetic nerve endings. $\beta 1$ receptors are localized in the heart and increase the heart rate and cardiac contractility, while $\beta 2$ receptors are mainly found in the lung and assist in ventilation by inducing bronchodilation.

Based on this information, the characteristics of the 3 types of oral medications discussed in this paper and their interactions with each other need to be re-evaluated.

$\beta$-blockers block $\beta$ receptors that are mostly located in the heart, reduce blood pressure, and generate a calming effect. However, surgeons need to bear in mind that the use of $\beta$ blockers can increase the sensitivity of $\alpha$ receptors. Normally, the epinephrine that is administered during surgery has greater affinity for $\beta$ receptors than for $\alpha$ receptors. However, if $\beta$ blockers are used preoperatively, when the epinephrine mixed in the local anesthetic is absorbed into the systemic circulation, it has a greater likelihood of binding to $a$ receptors. Overstimulation of $\alpha$ receptors leads to an ischemic condition of the myocardium, which is a situation that cardiologists or anesthesiologists take precautions against, and plastic surgeons are no exception to this.

In recent treatment regimens for high blood pressure, $\beta$ blockers are no longer considered the first-line medicine. In fact, if a patient with essential hypertension is taking $\beta$-blockers on a normal basis, it is most likely that his/her cardiologist pre- scribed the medication for stable angina, after a myocardial infarction, or for congestive heart failure. In such cases, epinephrine use is prohibited or $\beta$-blocker administration is temporarily stopped or replaced in advance with a different hypertension management drug with the consent and assistance of cardiologists or anesthesiologists.

In clinical practice, however, many surgeons prescribe a selective $\beta 1$-blocker (atenolol) to patients who normally do not have heart problems, use limited epinephrine, and perform outpatient aesthetic surgery using local anesthesia with hypnotics.

In contrast, $\alpha 2$ agonists inhibit the release of noradrenaline in the blood through negative feedback and lower the overall sympathetic tone, which has a more general calming effect and reduces blood pressure. Clonidine for oral medication and dexmedetomidine as an IV hypnotic are used in South Korea. As with other medications that react with the central nervous system structures that control negative feedback, when the administration of these medications is stopped, a rebound phenomenon (rebound hypertension) may occur.

However, this phenomenon is relative to the duration and dosage of the medication used for the patient. Therefore, rebound hypertension rarely occurs if clonidine is used in a single dose during the preoperative period. When the IV hypnotic dexmedetomidine is used, special attention is necessary because syncope caused by hypotension and bradycardia can occur, and patients should only be allowed to move or be discharged after a relatively long recovery time. It is safer and easier to monitor patients using clonidine (an oral medication) than dexmedetomidine (an IV hypnotic) when using $\alpha 2$ agonists.

Another problem with $\alpha 2$ agonist administration in South Korea is that most cardiologists and anesthesiologists have minimal clinical experience with these medications. Clonidine has an extremely limited use in pediatrics and psychiatry to treat tic disorders and attention-deficit/hyperactivity disorder. Neurologists most commonly prescribe dexmedetomidine to calm patients in intensive care units. Nevertheless, if the goal is to induce calm and reduce blood pressure by lowering the overall sympathetic tone during the perioperative period, these medications are ideal in theory.

If there is reluctance regarding $\beta$-blocker or $\alpha 2$ agonist administration because of patient-specific circumstances, CCBs can be an alternative. As they are one of the first-line medicines used for essential hypertension, cardiologists and anesthesiologists are familiar with CCBs, and CCBs reduce blood pressure relatively quickly. However, a significant disadvantage of CCBs is that they do not treat anxiety and tachycardia, which frequently occur in the context of surgery. When tachycardia occurs, shortacting $\beta$-blockers (esmolol) can control the situation, but as 
mentioned above, surgeons need to pay special attention to the dose of epinephrine and to the prevention of ischemic heart problems when administering $\beta$-blockers.

When a patient who has received preoperative antihypertensive medication enters the operating room and the IV line is secured, the waiting period for surgery needs to be short and immediate sedation should be induced to minimize anxiety in the patient. The IV hypnotics used for sedation-propofol, midazolam, ketamine, and dexmedetomidine-and their effect on the cardiovascular system is summarized in Table 2. In general, only ketamine stimulates the cardiopulmonary system, while the other 3 attenuate cardiopulmonary activity (Table 2).

The preferences of surgeons and the characteristics of patients determine whether these medications are used alone or in combination with each other. The decision-making in this situation should primarily aim to continuously maintain calmness in the patient and keep him/her from feeling anxiety or fear. The secondary goal should be to understand the effects that these medicines may have on the cardiovascular system and the respiratory system, and to be able to respond to changes by monitoring the patients.

Among the IV hypnotics, propofol is in many ways sufficient for facelift surgery because it most easily maintains sedation and does not cause postoperative nausea and vomiting. In our practice, we generally use propofol as the baseline hypnotic medication, and midazolam and ketamine as additive or combined medications in specific circumstances. However, we do not administer dexmedetomidine.

Surgeons should understand that the main cause of perioperative hypertension is an anxiety-induced increase in the sympathetic tone in the autonomic nervous system in order to keep the blood pressure within the normal range during the perioperative period. Simply thinking that he/she can control hemorrhage during the intraoperative period or that he/she can prevent hematoma formation with a compression dressing (appli- cation of negative suction drainage or an elastic bandage) in the postoperative period is close to a delusional belief that he/she can solve the patient's anxiety with electrocautery burns or compression dressing. Lastly, perioperative hypertension management is a significant clinical process that can make a considerable difference in the patient's recovery. Moreover, at the time of surgery, in the absence of cardiologists or anesthesiologists, addressing any anesthesia-induced cardiovascular concerns will be the surgeon's responsibility.

\section{REFERENCES}

1. Varon J, Marik PE. Perioperative hypertension management. Vasc Health Risk Manag 2008;4:615-27.

2. Trussler AP, Hatef DA, Rohrich RJ. Management of hypertension in the facelift patient: results of a national consensus survey. Aesthet Surg J 2011;31:493-500.

3. Grover R, Jones BM, Waterhouse N. The prevention of haematoma following rhytidectomy: a review of 1078 consecutive facelifts. Br J Plast Surg 2001;54:481-6.

4. Matarasso A, Elkwood A, Rankin M, et al. National plastic surgery survey: face lift techniques and complications. Plast Reconstr Surg 2000;106:1185-95.

5. Mathes SJ, Hentz VR. Plastic surgery. Vol. I, General principles. Philadelphia, PA: Saunders Elsevier; 2006.

6. Neligan PC, Gurtner GC, Elsevier. Plastic surgery. Vol. 1, Principles. London: Elsevier/Saunders; 2013.

7. Beninger FG, Pritchard SJ. Clonidine in the management of blood pressure during rhytidectomy. Aesthet Surg J 1998; 18:89-94.

8. Neligan PC. Plastic surgery. Vol. 2, Aesthetic. London: Elsevier Saunders; 2013.

9. Jones BM, Grover R. Avoiding hematoma in cervicofacial rhytidectomy: a personal 8-year quest. Reviewing 910 patients. Plast Reconstr Surg 2004;113:381-7. 